\title{
Forming pressure effect on microstructure and mechanical properties of nanocrystalline aluminum synthesized by inert gas condensation
}

\author{
Shangshu Wu, Zhou Yu, Junjie Wang, Hanxin Zhang, Chaoqun Pei, Shu Fu, Hao Fu, Jiajie Meng and Tao Feng* \\ Herbert Gleiter Institute of Nanoscience, Nanjing University of Science \& Technology, Nanjing, People's Republic of China
}

\begin{abstract}
The preparation of nanocrystalline aluminum ( $\mathrm{NC} \mathrm{Al}$ ) was conducted in two steps. After the NC $\mathrm{Al}$ powder was synthesized by an Inert gas condensation (IGC) method in a helium atmosphere of $500 \mathrm{~Pa}$, the NC Al powder was in-situ compacted into a pellet with a $10 \mathrm{~mm}$ diameter and $250 \mu \mathrm{m}-300 \mu \mathrm{m}$ thickness in a high vacuum $\left(10^{-6} \mathrm{~Pa}-10^{-7} \mathrm{~Pa}\right)$ at room temperature. The $\mathrm{NC} \mathrm{Al} \mathrm{samples} \mathrm{were} \mathrm{not} \mathrm{exposed} \mathrm{to} \mathrm{air} \mathrm{during}$ the entire process. After the pressure reached $6 \mathrm{GPa}$, the relative density could reach $99.83 \%$. The results showed that the grain size decreased with the increased of in-situ forming pressure. The $\mathrm{NC} \mathrm{Al} \mathrm{samples}$ present obvious ductile fracture, and the tensile properties were greatly changed with the increase of forming pressure.
\end{abstract}

\section{Introduction}

Aluminum and aluminum alloys play an important role in the aerospace and automotive industries owing to the excellent property, light in weight and resistant to corrosion. Therefore, research on aluminum has important practical significance[1]. The motivation for research interest in bulk nanostructured materials attributed to the excellent work of H.Gleiter and his Partners in the early 1980s. They first prepared bulk nanostructured materials by inert gas condensation (IGC) and found that their microstructure and properties are considerable different from traditional coarse-grained materials[2-4]. While changing the structure of the material, the strength as well as hardness of the material are improved $[5,6]$. For nanocrystalline materials, a large number of interfacial structures can be introduced by reducing the size of the crystal grains to the nanometer magnitude. Due to the different arrangement of atoms at the interface and inside the grain, they show different properties[7]. The relevant properties of the material can be tuned by the introduced interface defect. A recent study by F. Tang and cooperators[8] explained the deformation mechanism of thin film nanocrystalline aluminum ( $\mathrm{NC} \mathrm{Al}$ ) prepared under different vacuum degrees. The variation of interfacial oxygen content is used as a variate to explain the effect of impurities on the mechanical properties and thermal stability of $\mathrm{NC} \mathrm{Al}$. The performance is proportional to the oxygen content, which is also suitable for thermal stability. M. Abou Zied et al. found that effect of peaks expansion at different pressures on the structure of nanocrystals in XRD test[9]. Under different pressures, the lattice constant of the sample shows a regular change, and the pressure has a significant influence on the internal microstructure of the $\mathrm{NC} \mathrm{Al}$. High grain size stability of NC Al was introduced by F. Zhou. J. Lee found the thermal stability of $\mathrm{NC} \mathrm{Al}$ of different grain sizes[10]. The thermal stability of $\mathrm{NC} \mathrm{Al}$ can reach $0.78 \mathrm{Tm}$. X. Z. Liao et al $[5,11]$ showed the wide stacking faults formation mechanism and deformation twins in $\mathrm{NC} \mathrm{Al}$, a more profound explanation of the microscopic deformation mechanism of $\mathrm{NC} \mathrm{Al}$ has been made. The grain size plays a crucial role in understanding the deformation mechanism of $\mathrm{NC}$ Al.

The preparation process of the material determines the microstructure of the sample, which affects the properties of the material. It is well known that the current methods for preparing bulk nanomaterials can be roughly divided into two categories: bottom-up and top-down. The inert gas condensation method is a typical bottom-up method, which structurally reorganizes metal materials from the atomic dimension, and is the most advantageous method for obtaining bulk nanomaterials at the current. In addition, the nanocrystalline material prepared by the inert gas condensation method is no longer limited to the thin film, and is more favorable for the study of the structure and properties of the bulk nanocrystalline materials.

In this work, $\mathrm{NC} \mathrm{Al} \mathrm{was} \mathrm{prepared} \mathrm{by} \mathrm{IGC} \mathrm{method,}$ and the in-situ forming pressure effect on the microstructure and mechanical properties of the $\mathrm{NC} \mathrm{Al}$ was systematically studied. Under ultra-high pressure, the porosity of $\mathrm{NC} \mathrm{Al}$ was reduced dramatically, and the density could reach $99.83 \%$ of the coarse grain Al. The microstructure of $\mathrm{NC} \mathrm{Al}$ with different forming pressure were characterized by XRD, SEM, and TEM, and the mechanical properties were studied by the tensile test.

* Corresponding author: tao.feng@njust.edu.cn 


\section{Experimental procedure}

The raw material was a commercially pure $\mathrm{Al}$ powder (purity $\geq 99.9 \%$ ), which was purchased from Ningbo Shanshan New Material Technology Co., Ltd. The NC Al was synthesized by an IGC method. As shown in the Fig. 1. (a) and (b), our IGC system consists mainly of three units, powder preparation unit, low pressure compaction unit, and high pressure compaction unit. At the first step, in the powder preparation unit, the raw material Al was evaporated in the helium (500 $\mathrm{Pa}$ ) when the temperature reached $1400^{\circ} \mathrm{C}$. $\mathrm{Al}$ atoms lost kinetic energy and condensed in the form of small crystals a few nanometers across, which accumulate on a vertical cold finger as a loose powder. The NC Al powder was stripped off and collected into a container. At the second step, the $\mathrm{NC} \mathrm{Al}$ powder was transferred to the low pressure compaction unit and compressed into a pellet with $10 \mathrm{~mm}$ diameter under the pressure of $500 \mathrm{MPa}$. Finally, the pellet was transferred to the high pressure compaction unit to condense under the pressure of 2-6 GPa. Our NC Al sample is formed by pressure only, without any heat treatment.

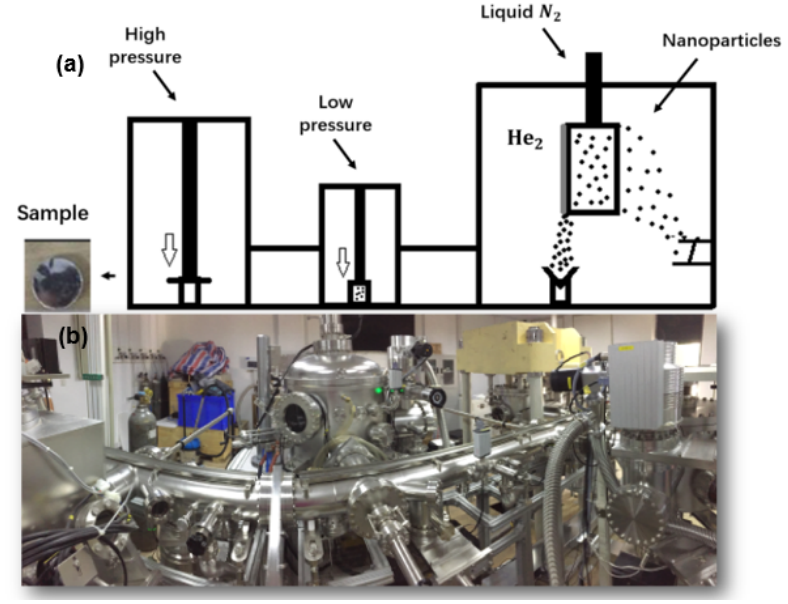

Figure. 1. (a) the schematic diagram and (b) the photo of inert gas condensation (IGC) system.

The grain size of the $\mathrm{NC} \mathrm{Al}$ powder and bulk NC Al sample were determined by X-ray diffraction (XRD) analysis and transmission electron microscope (TEM) observation. The XRD testing was carried out on a Bruker-AXS D8 Advance diffractometer with $\mathrm{Cu} \mathrm{K \alpha}$ radiation. Microstructures were characterized by a FEI Tecnai 20 (TECNAI G2 20 LaB6) transmission electron microscopy. The TEM powder specimen was prepared by dispersing $\mathrm{NC} \mathrm{Al}$ powder in alcohol anddripping onto the TEM copper grid after ultrasounding for $20 \mathrm{~min}$. The TEM foils specimen was prepeard by mechanically polishing the $\mathrm{NC} \mathrm{Al}$ pellet to the thickness of $50 \mu \mathrm{m}$ and then cutting into a circle with diameter of $3 \mathrm{~mm}$, finally corroding in methanol mixed with nitric acid (7:3) solution. The density of NC Al samples was measured by volume-weight method (Archimedes principle). The tensile tests were conducted on instron E3000 tensile machine at room temperature, with a constant strain rate
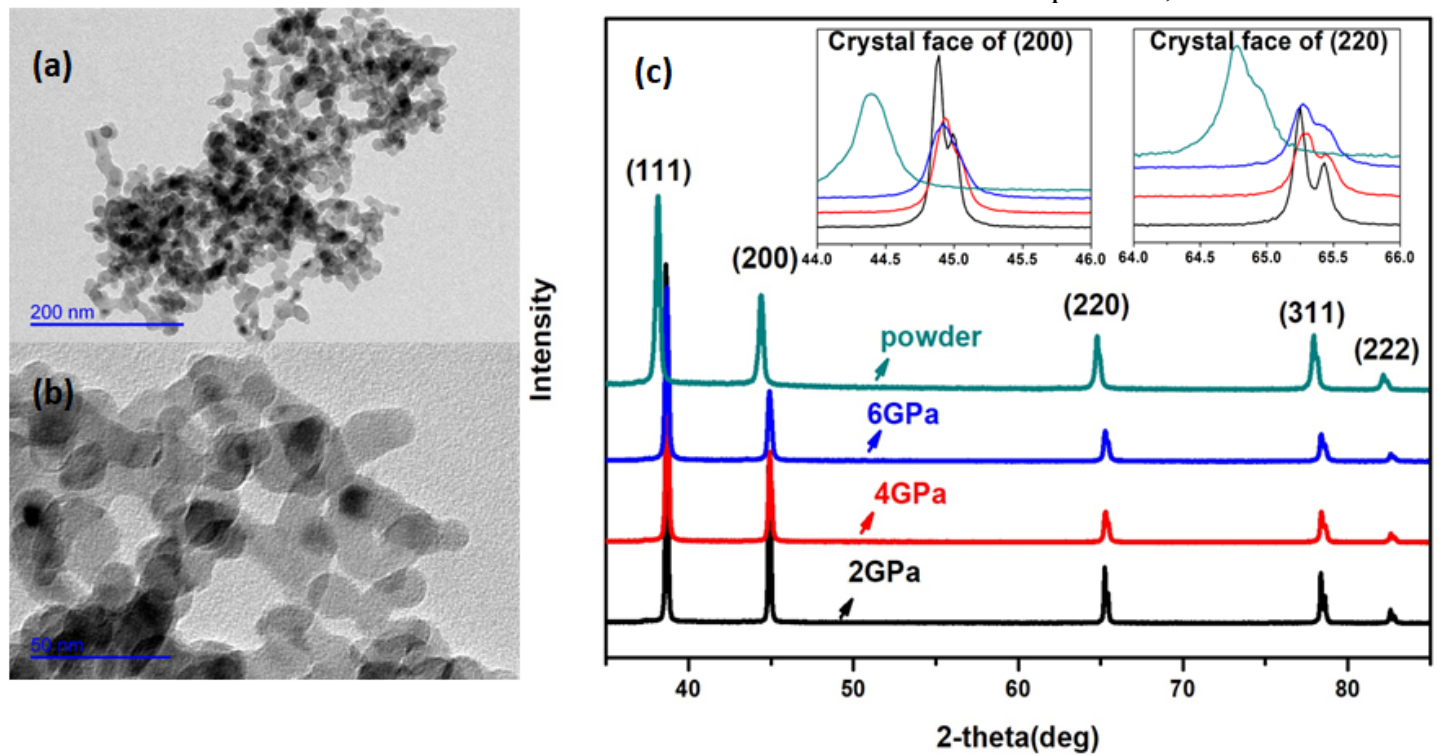

Figure. 2. (a) and (b) are the TEM images of the as-prepared $\mathrm{NC}$ Al powder synthesized by inert gas condensation, (c) XRD patterns of $\mathrm{NC} \mathrm{Al} \mathrm{powder} \mathrm{and} \mathrm{pellets} \mathrm{under} \mathrm{different} \mathrm{forming} \mathrm{pressure.}$

Table 1. Structural parameters under different pressures

\begin{tabular}{|c|c|c|c|c|c|}
\hline sample & $(200) / \theta^{\circ}$ & $(220) / \theta^{\circ}$ & $\begin{array}{c}\text { lattice } \\
\text { constant } \\
/ \AA\end{array}$ & $\begin{array}{l}\text { interplanar distance } \\
\quad \text { of }(200) / \AA\end{array}$ & $\begin{array}{l}\text { interplanar distance } \\
\text { of }(220) / \AA\end{array}$ \\
\hline
\end{tabular}




\begin{tabular}{rrrrcr}
\hline $2 \mathrm{GPa}$ & 44.882 & 65.247 & 4.04171 & 2.020855 & 1.42896 \\
$4 \mathrm{GPa}$ & 44.886 & 65.256 & 4.04063 & 2.020315 & 1.42858 \\
$6 \mathrm{GPa}$ & 44.909 & 65.263 & 4.0405 & 2.02025 & 1.42853 \\
\hline
\end{tabular}

of $1 \times 10^{-4} \mathrm{~s}^{-1}$. Crosshead displacement was used to calculate strain, the initial sample cross section was used to calculate stress, and the gage length after failure was used to calculate the elongation to failure of the sample.

The observation of the tensile-specimen fractures was made on a FEI Quanta 250F scanning electron microscope (SEM).

\section{Results and discussion}

Fig.2. (a) and (b) are the transmission electron micrograph of the NC Al powder. Dense alumina film appeared on the surface of the $\mathrm{NC} \mathrm{Al}$ powder and prevented the further oxidation after the powder was taken out from the chamber. A statistical analysis of 500 grains showed an average particle size of $25 \mathrm{~nm}$. The results of grain size tested by the TEM is consistent with the XRD calculation. Fig.2. (c) is a crystal plane diffraction peaks of NC Al. Firstly, the diffraction peaks of the two crystal faces of (200) and (220) are smoothly taken. The changes of the diffraction peak parameters under different forming pressures can be used to obtain the change of $\mathrm{NC} \mathrm{Al}$ crystal structure after in-situ high

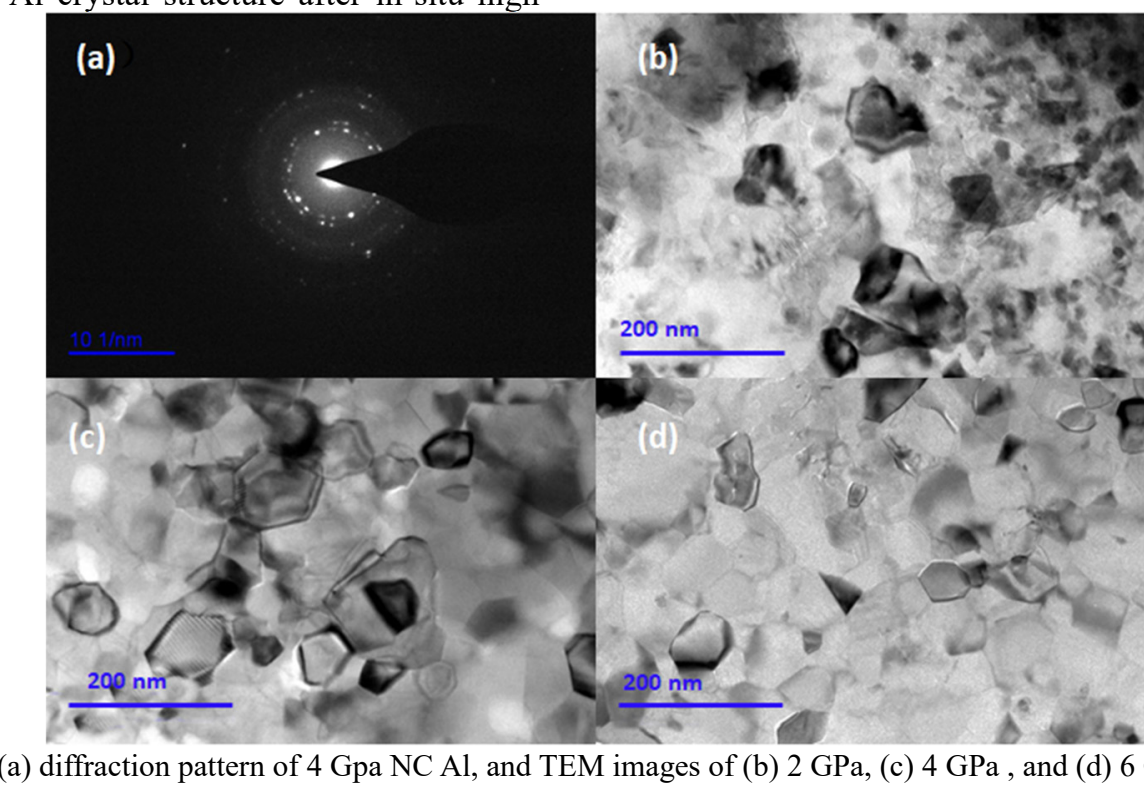
obtained.

Figure. 3. (a) diffraction pattern of $4 \mathrm{Gpa} \mathrm{NC} \mathrm{Al}$, and TEM images of (b) $2 \mathrm{GPa}$, (c) $4 \mathrm{GPa}$, and (d) $6 \mathrm{GPa} \mathrm{NC} \mathrm{A}$

pressure compaction. The lattice constant is a basic parameter reflecting the structural size of the crystalline material, which directly reflects the binding energy between the particles. The magnitude of the change in lattice constant is small (about $10^{-5} \mathrm{~nm}$ ). The lattice constant a can be calculated using the Bragg equation and the interplanar spacing formula. $\mathrm{NC} \mathrm{Al} \mathrm{belongs} \mathrm{to}$ the face-centered cubic:

$$
=\frac{\lambda \sin \theta=\mathrm{n} \lambda}{2 \sin \theta} \sqrt{h^{2}+k^{2}+l^{2}}
$$

Where, $\lambda$ is the incident $\mathrm{X}$-ray wavelength; $\mathrm{n}$ is the diffraction order; $\theta$ is the Bragg angle. The deviation in the measurement of the lattice constant is mainly derived from the wavelength $\lambda$, $\sin \theta$ and the interference surface index (h, k, l). $\mathrm{n}$ is usually taken as $1, \lambda$ is $0.154056 \mathrm{~nm}$, and $\mathrm{h}, \mathrm{k}$, and $\mathrm{l}$ are integers, so the source of the lattice constant deviation is mainly $\sin \theta$. The lattice constant can be obtained by bringing the parameters of Table 1 into the above formula. Bringing the obtained lattice constant into the formula of the interplanar spacing of the cubic crystal system, the interplanar spacing can be

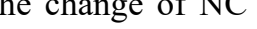

$$
\mathrm{d}=\frac{a}{\sqrt{h^{2}+k^{2}+l^{2}}}
$$

From the above results, it can be seen that as the in-situ pressure increases from $2 \mathrm{GPa}$ to $6 \mathrm{GPa}$, the lattice constant decreases from $4.04171 \AA$ to $4.04063 \AA$ and then decreases to $4.0405 \AA$. It is owing to vacancies' formation[12]. It is also accompanied by a decrease in the interplanar spacing. The variation of the interplanar spacing is reduced from $2.020855 \AA$ of the (200) crystal plane to $2.02025 \AA$, and the $(220)$ crystal plane is reduced from $1.42896 \AA$ to $1.42853 \AA$

In addition, the influence of pressure on the grain size change can also be analyzed from the X-ray diffraction pattern. The larger the full width at half maximum (half width) of the diffraction peak on the diffraction curve,

the smaller the grain size. The grain size of the constituent material can be expressed by the full width at half maximum in the X-ray diffraction region. From the Fig. 2. (c) can be seen that as the molding in-situ pressure increases, significant peak broadening occurs, peak broadening and grain size closely related. Use the Scherrer formula can get the grain size:

$$
\mathrm{D}_{h k l}=\frac{0.89 \lambda}{\beta \cos \theta}
$$

Where, $D_{h k l}$ is the grain size perpendicular to the $(\mathrm{hkl})$ crystal plane; $\beta$ is the half-height width of the diffraction peak due to microcrystal refinement. It can be seen from the measurement results that on the diffraction curve, the full width at half maximum of the (200) and (220) crystal plane diffraction peaks increased as the in-situ pressure increased. According to the Scherrer formula, with the 
in-situ pressure increased, the grain size perpendicular to the (220) and (200) crystal planes gradually decreased.

Table 2. Grain size and density

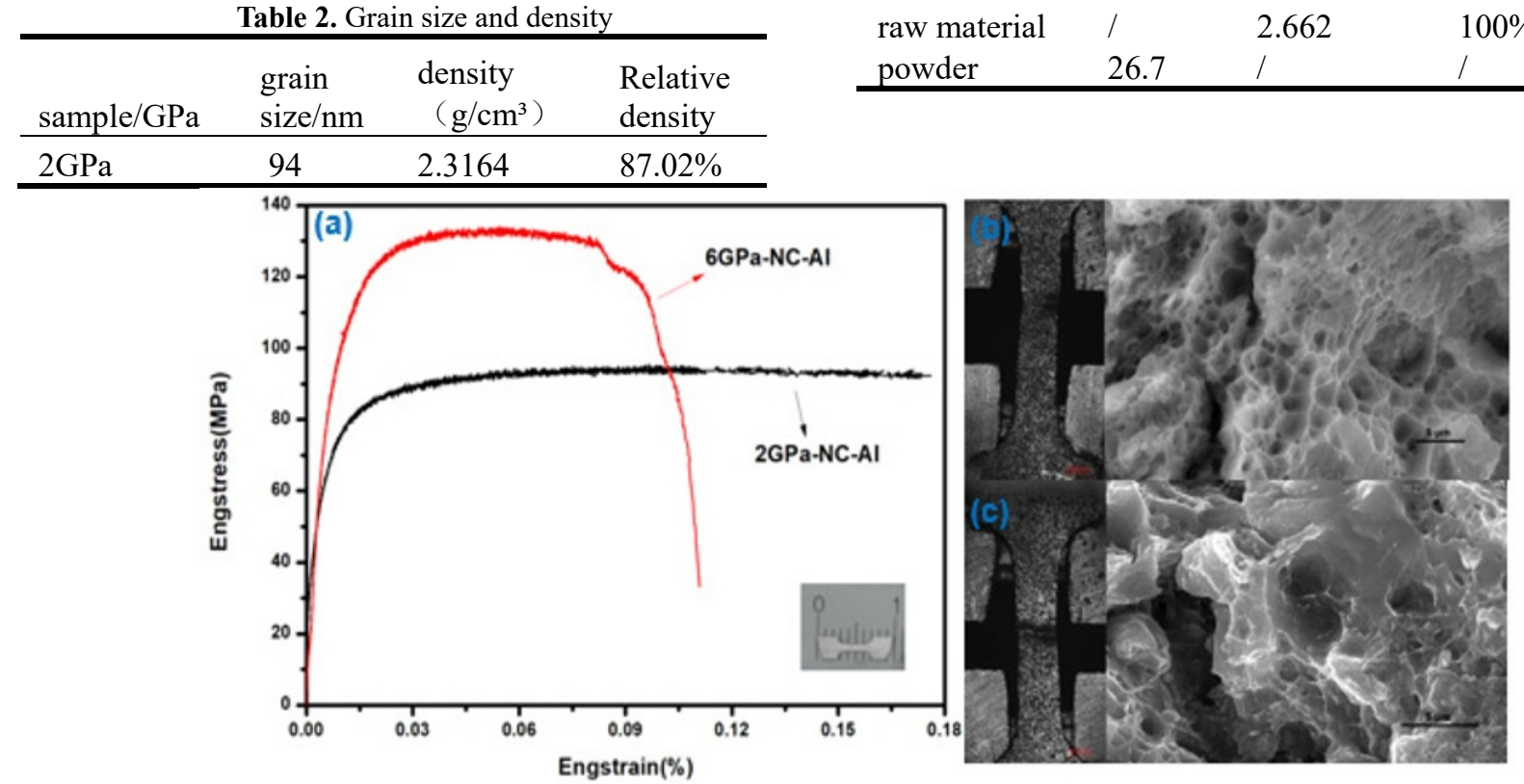

Figure. 4. (a) tensile curve of $2 \mathrm{GPa}$ and $6 \mathrm{GPa} \mathrm{NC} \mathrm{Al} \mathrm{samples,} \mathrm{(b)} \mathrm{and} \mathrm{(c)} \mathrm{fractured} \mathrm{surface} \mathrm{of} \mathrm{tensile} \mathrm{specimens} \mathrm{with} 2$ GPa and 6 $\mathrm{GPa}$, respecitively.

Sanders et al., [13] Ma., [14] and Youngdahl et al[15] have studied the effects of processing defects on nanocrystalline materials on mechanical properties. They showed the results that the processing defects would reduce hardness, strength, and plasticity. All of the processing defects may lead to untrustworthy information, mainly about mechanical properties. The current work also studies the effects of processing defects on the mechanical properties of samples. As the forming pressure increased from $2 \mathrm{GPa}$ to $6 \mathrm{GPa}$, the grain size of the $\mathrm{NC} \mathrm{Al} \mathrm{sample} \mathrm{decreased} \mathrm{from} 94 \mathrm{~nm}$ to $45 \mathrm{~nm}$. At the same time, the relative density of $6 \mathrm{GPa}$ sample can reach $99.83 \%$ of coarse grain Al. Fig. 3. (a) is the diffraction pattern of $4 \mathrm{GPa}$ sample, it shows clear polycrystalline features. Fig. 3. (b) (c) and (d) show the grain size of $\mathrm{NC} \mathrm{Al}$, and it corresponds to the XRD result. Generally, the strength of polycrystals generally obeys the Hall-Petch relationship as the grain size changes[16].

$$
\sigma=\sigma_{0}+\mathrm{kd}^{-1 / 2}
$$

$\sigma_{0}$ is the intensity constant; $\mathrm{k}$ is the normal number; $\mathrm{d}$ is the grain size. According to the Hall-Petch empirical formula, the strength of the material is inversely related to the grain size of the material. For traditional coarse crystals, the deformation mechanism is the interaction of unit dislocations and the work hardening control plasticity. For nanocrystals, as the grain size decreases to the nanometer level, a large number of interfaces are introduced to produce high-density grain boundaries. Grain boundaries hinder the dislocation movement which causes the increasement of hardness and strength of the material. The deformation mechanism of the material becomes that dislocations and dislocation stacks emit from grain boundaries and promote grain boundaries to shear. However, when the grain size of the material is less than $10 \mathrm{~nm}$, grain boundary sliding and grain rotation become the dominant deformation mechanism, and an abnormal Hall-Petch relationship occurs[17-24].

As shown in the Fig. 4. (a), comparing the tensile properties of the $\mathrm{NC} \mathrm{Al}$ samples under the forming pressure of $2 \mathrm{GPa}$ and $6 \mathrm{GPa}$, the obtained results are consistent with the Hall-Petch relationship. The tensile strength of the $\mathrm{NC} \mathrm{Al}$ increased from $94 \mathrm{MPa}$ of $2 \mathrm{GPa}$ samples to $134 \mathrm{MPa}$ of $6 \mathrm{GPa}$ samples, which is $40 \%$ increasement. The necking phenomena were observed during the tensile test for both kinds of NC Al. Fig. 4. (b) and (c) are the SEM photographs of the fractures of the two samples with a large number of dimples, which shows the obvious ductile fracture feature.

\section{Conclusions}

In this work, pure NC Al was prepared by IGC method under different in-situ forming pressure from $2 \mathrm{GPa}$ to 6 GPa. The microstructure and mechanical preoperties were systematically studied by XRD, SEM, TEM and tensile test. When the pressure reached $6 \mathrm{GPa}$, the relative density of the NC Al sample could reach $99.83 \%$. With the forming pressure increase, the lattice constant and crystal surface spacing of the samples showed regular changes. The grain size decreased from $94 \mathrm{~nm}$ to $45 \mathrm{~nm}$, and the tensile strength increased from $94 \mathrm{MPa}$. The tensile strength of $6 \mathrm{GPa}$ sample increased by $40 \%$ comparing to $2 \mathrm{GPa}$ sample. All the $\mathrm{NC} \mathrm{Al}$ samples showed obvious ductile fracture.

\section{Acknowledgment}

This work was supported by the National Natural Science Foundation of China (grant no. 51571119) and the Fundamental Research Funds for the Central Universities (No. 30916011106). TF acknowledges the support by the innovation project, Qing Lan project and 
the specially-appointed professor project of Jiangsu province.

\section{References}

1. I. Matsui, S. Ono, Y. Takigawa, T. Uesugi, K. Higashi. Mater. Sci. Eng. A 550, 363 (2012)

2. H. Gleiter. Prog. Mater. Sci 33, 223 (1989)

3. R. Birringer, H. Gleiter, H.P. Klein. Phys. Lett. A 41, 1033 (1993)

4. H. Gleiter, P. Marquardt, Z. Metallkunde 75, 263 (1984)

5. X.Z. Liao, F. Zhou, E.J. Lavernia. Appl. Phys. Letters 83, 5062 (2003)

6. M.A. Meyers, A. Misher, D.J. Benson. Prog. Mater. Sci 51, 427 (2006)

7. P.L. Sun, E.K. Cerreta, J.F. Bingert, G.T. Gray. Mater. Sci. Eng. A 464, 343 (2007)

8. F. Tang, D.S. Gianola, M.P. Moody, K.J. Hemker, J.M. Cairney. Acta. Mater 60, 1038 (2012)

9. M.A. Zied, A.A Ebnalwaled. Intermetallics 16, 745 (2008)

10. F. Zhou, J. Lee. J Mater Res 16, 12 (2001)

11. X.Z. Liao, S.G. Srinivasan, Y.H. Zhao, M.I. Baskes, Y.T. Zhu, F. Zhou, et al. Appl. Phys. Letters 84, 3564 (2004)

12. R.A. Varin, J. Bystrzycki, A. Calka. Intermetallics 7, 917 (1999)

13. P.G. Sanders, C.J. Youngdahl, J.R. Weertman. Mater. Sci. Eng 234, 77 (1997)

14. L. He, E. Ma. J. Mater. Res 11, 72 (1996)

15. C.J. Youngdahl, P.G. Sanders, J.A. Eastman, J.R. Weertman. Scripta. Mater 37, 809 (1997)

16. G.D. Hughes, S.D. Smith, C.S. Pande. Scripta. Metall. Mater 20, 93 (1986)

17. C.Y. Yu, P.W. Kao, C.P. Chang. Acta. Mater 53, 4019 (2005)

18. H. Gleiter. Adv Mater 4, 474 (1992)

19. S. Cheng, E. Ma, Y.M. Wang, et al. Acta. Mater 53, 1521 (2005)

20. Y.M. Wang, E. Ma. Acta. Mater 52, 1699 (2004)

21. H. Conrad, Mater. Sci. Eng. A 341, 216 (2003)

22. K.S. Kumar, H.Van. Swygenhoven. S. Suresh. Acta. Mater 51, 5743 (2003)

23. E.O. Hall. Proc. Soc. London 64, 747 (1951)

24. N.J. Petch. Iron Steel Inst 174, 51 (2006) 